\title{
Effect of Tip Morphology of Vertically Aligned Alumina Nanowire Arrays on Ovalbumin Uptake of Dendritic Cells
}

\author{
Shree C. Aier ${ }^{1}$, Kavita Meduri ${ }^{1}$, Matthew Newman ${ }^{2}$, Robin Ekeya ${ }^{1}$, Patrick Crawford ${ }^{1}$, Dustin Austin ${ }^{3}$, \\ Lester Lampert ${ }^{1}$, John F. Conley Jr. ${ }^{3}$ Evan Lind ${ }^{2}$, Jun Jiao ${ }^{1}$ \\ 1. Mechanical and Materials Engineering, Portland State University, Portland, OR. USA. \\ 2. Molecular Microbiology \& Immunology, Cell and Developmental \& Cancer Biology, Oregon Health \\ \& Science University, Portland, OR. USA. \\ 3. Electrical Engineering, Materials Science, Oregon State University, Corvallis, OR. USA.
}

Previous research on nanoscale protein delivery systems has demonstrated great potential for their use in cancer research [1]. Here, we have studied structural variations in tip morphology of nanowires (NWs), and have investigated the impact of these structural variations on the resultant protein delivery. Vertically aligned carbon nanotubes (CNTs) were grown by plasma enhanced chemical vapor deposition (PEVCD), and coated with a $25 \mathrm{~nm}$ alumina coating [2]. The fabricated vertically aligned alumina nanowire arrays (VAANAs) were used to deliver fluorescent Alexa Fluor 488 conjugated to ovalbumin protein (OVAC) to primary dendritic cells (DCs) derived from murine bone marrow.

Scanning electron microscopy (SEM) was used to categorize VAANAs with observed structural morphological differences (Figure 1). VAANA Type I was defined as narrow NWs that clumped together and exhibited tapered ends. VAANA Type II was defined as uniform NWs with distinct individual tips. Both types were used in OVAC delivery to primary DCs. OVAC were anchored onto the VAANAs using the chemical linker (3-Aminopropyl) trimethoxysilane (APTMS) [1]. Next excess OVAC was removed, and DCs were then cultured onto the VAANAs. After three hours of incubation, cells were further stained with a live/dead cell assay with Zombie Red. The DCs were subsequently fixed with $4 \%$ paraformaldehyde. Flow cytometry analysis with 100,000 cells was conducted on BD Fortessa.

Type I and II VAANAs were compared for their OVAC delivery efficiency to a baseline solution based delivery system, where the same concentration of OVAC was directly added to the cell media. Approximately $90 \%$ of DCs trypsinized (removed) from type I and II VAANAs were deemed viable by the Zombie Red assay. Figure 2a shows a sample gating strategy, and the results in Figure $2 \mathrm{~b}$ indicate that VAANA type II (individual tips) produced a significantly higher (143\%) OVAC delivery when compared to the solution-based system (baseline). However, VAANA type I (tapered ends) resulted in lower delivery (38\% less) than the baseline. Additionally, after cells were removed (trypsinized) from the two samples, the VAANAs were analyzed by spinning disk confocal microscopy to qualitatively compare the amount of OVAC remaining/anchored on the NWs. Confocal imaging results (Figure 3) were consistent with those of described in Figure 1 and 2. Note in Figure 3 that there was a significantly higher amount of OVAC remaining on the surface of VAANA type I than type II. This difference demonstrates that DCs ingested more protein when interacting with VAANA type II.

Results suggest that minor structural morphology may play a significant role in efficient protein delivery, despite using the same recipe for fabrication of VAANAs. One possible physical basis for these data may be the observed difference in structural rigidity and number of contact points between type I and II. While OVAC delivery is significantly higher for VAANA type II, further analysis should 
be completed to assess differences in functional maturation markers, such as major histocompatibility complexes (MHC), for effective usage with DCs and cancer research. A follow up paper will be published to elucidate these variances.

\section{References:}

[1] Shalek A. et al. PNAS 107 (2009), p. 1870.

[2] Lampert L. et al, Microsc. Microanal. 30 (2014) p.1972.

[3] The research is funded in part by EXITO grant from the NIH and NSF/REU grant (No. 1560383).
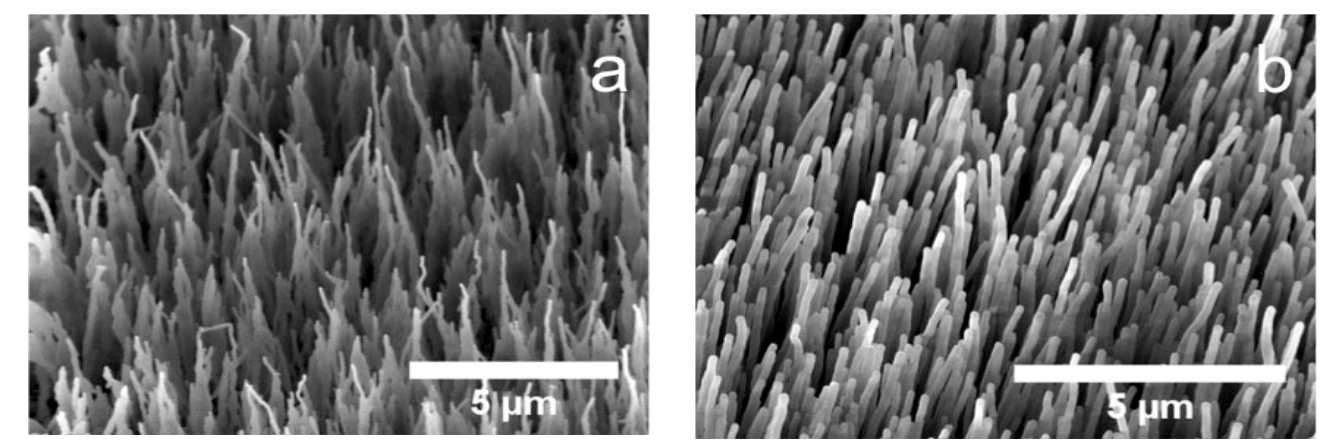

Figure 1: Two types of VAANAs with different morphologies were used in the study. (a) VAANAs with tapered tips type I. (b) VAANAs with individual tips type II.
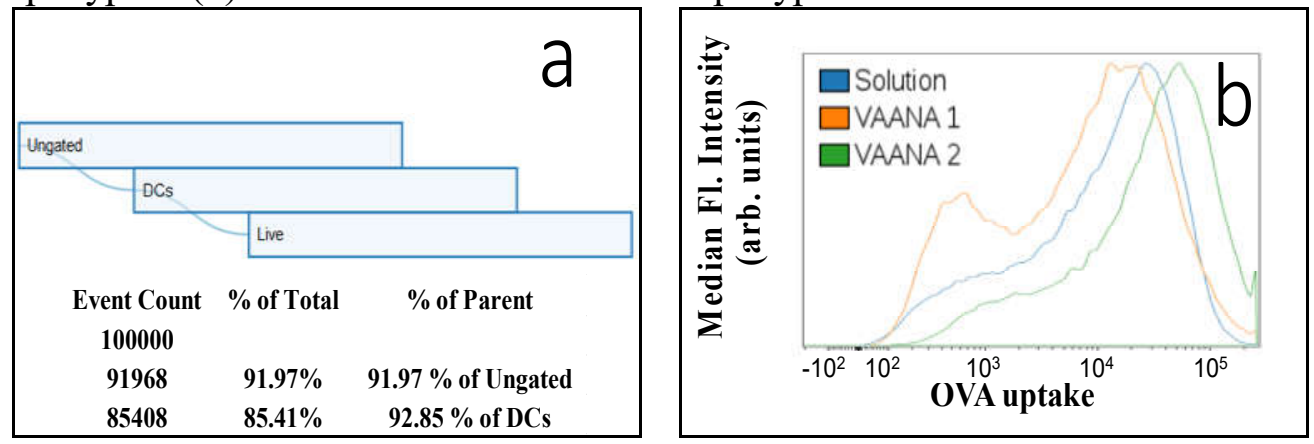

Figure 2: Flow cytometry analysis. (a): Gating Strategy. CD11c and CD11b differentiation markers were used to sort cells. From these, live cells were used for further analysis using Zombie Red. (b): VAANA II produced significantly higher median fluorescence intensity (143\%) indicating greater uptake of OVAC.
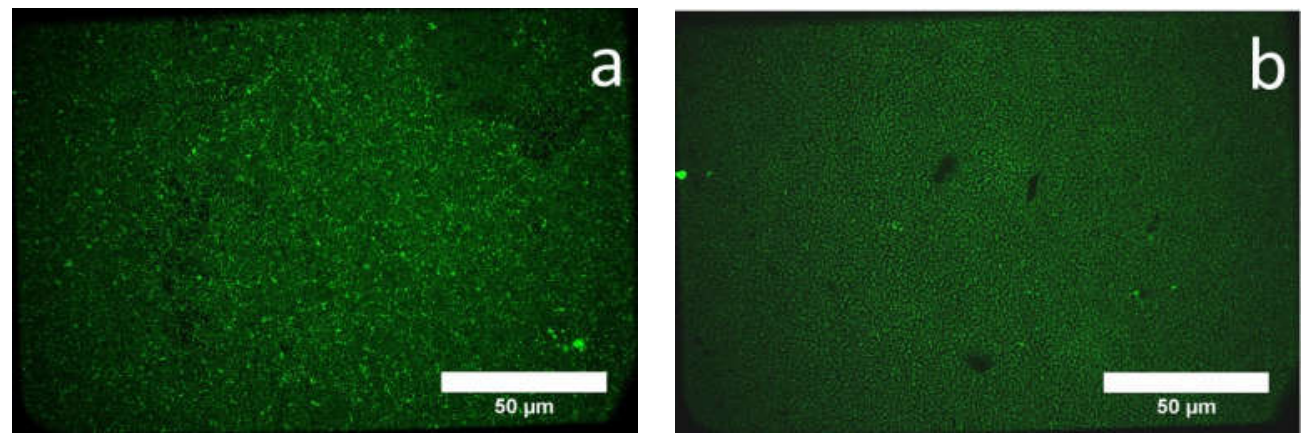

Figure 3: Confocal images of VAANA I (a) and II (b) taken after removal of cultured cells. Images confirm that DCs were able to ingest more OVAC from VAANA II (b) (less fluorescence indicates less remnant OVAC). VAANA type I (a) showed higher fluorescence indicating higher residual OVAC. 